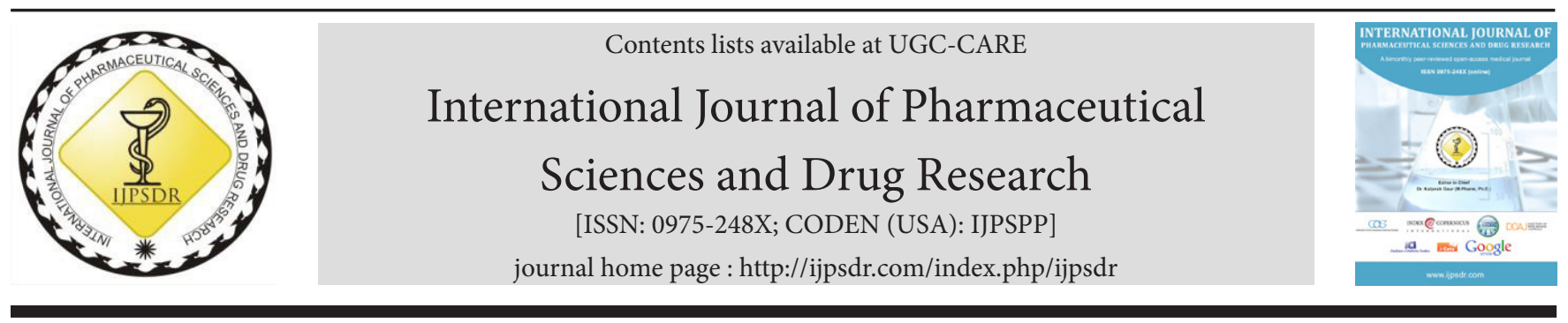

Research Article

\title{
Design, Development, and Delivery of Rasagiline mesylate from Monolithic Drug in Adhesive Matrix Patches
}

\author{
Vipulbhai Mandli $^{1 *}$, Shailesh T. Prajapati ${ }^{2}$ \\ ${ }^{1}$ Department of Pharmaceutical Sciences, Hemchandracharya North Gujarat University, Patan-384265, Gujarat, India \\ ${ }^{2}$ Department of Pharmaceutics and Pharmaceutical Technology, Kalol Institute of Pharmacy KIRC Campus, Ahmedabad-Mehsana Highway, Kalol, \\ Gandhinagar-384265, Gujarat, India
}

\begin{tabular}{l} 
A R T I C L E I N F O \\
\hline Article history: \\
Received: 28 November, 2019 \\
Revised: 08 January, 2020 \\
Accepted: 10 January, 2020 \\
Published: 30 January, 2020 \\
Keywords: \\
Backing film, \\
Cold flow, \\
Durotak-4098, \\
Ex-vivo percutaneous absorption, \\
GSM, In-vitro adhesion testing, \\
Rasagiline Mesylate, \\
Release liner. \\
D0I: \\
10.25004/IJPSDR.2020.120111
\end{tabular}

\section{INTRODUCTION}

A transdermal system (TDS) is proposed to release the medicine into systemic circulation through the skin to cure disorders in locations far away of the site of application. Exact shape and size of the TDS are available for systemic action and are proposed for the treatment or prevention of systemic disease. Medicine released from the TDS is absorbed through skin into blood circulation and reached to target tissues to achieve a therapeutic effect. ${ }^{[1]}$ The TDS has many merits over conventional

\begin{abstract}
A B S T R A C T
The purpose of this research was to prepare and evaluate monolithic drug-in-adhesive type patches of rasagiline mesylate (RM) containing penetration enhancer and having 7-day wear property. Preformulation studies like solubility in permeation enhancers, compatibility study, transmission study, uptake study, and crystallization study of RM in various pressure-sensitive adhesive (PSA) polymers were performed. The transdermal system was prepared by the solvent casting method. The effects of various permeation enhancers (Propylene glycol, oleic acid, isopropyl palmitate, and lauryl lactate) on the ex-vivo transcutaneous absorption of RM through human cadaver skin were evaluated by modified Franz diffusion cell system. Ex-vivo transcutaneous absorption of the prepared transdermal patch was performed using different concentrations of Lauryl lactate (3, 5, and 7\%). In-vitro Adhesion testing (Peel, tack shear, etc.) was performed on different dry grams per square meter (GSM) of patches like 80GSM, $100 \mathrm{GSM}$, and 150 GSM. The final transdermal patches were tested for appearance, the weight of matrix, thickness, \% assay of drug content, in-vitro adhesion testing, cold flow study, and ex-vivo skin permeation studies. Based on crystallization study and adhesion testing, Durotak-4098 (14\% drug concentration) was selected as a PSA. A patch containing Lauryl lactate showed the highest cumulative permeation compared to other permeation enhancers. The patch containing $5 \%$ lactate showed greater transdermal flux $\left(2.36 \mu \mathrm{g} / \mathrm{cm}^{2} /\right.$ hr). Patch with 150 dry GSM showing promising adhesion properties. Backing film Scotchpak 9723 and release liner Saint Gobain 8310 was selected based on transmission and uptake study of RM. The stability study indicates that developed formulation remains stable. In conclusion, the present research confirms the practicability of developing a RM transdermal system.
\end{abstract}

\footnotetext{
*Corresponding Author: Mr. Vipulbhai Mandli

Address: Department of Pharmaceutical Sciences, Hemchandracharya North Gujarat University, Patan-384265, Gujarat, India

Email $\bowtie$ : vipul_mandli@yahoo.com

Tel.: +91-9898146888

Relevant conflicts of interest/financial disclosures: The authors declare that the research was conducted in the absence of any commercial or financial relationships that could be construed as a potential conflict of interest.

Copyright (C) 2020 Vipulbhai Mandli et al. This is an open access article distributed under the terms of the Creative Commons AttributionNonCommercial-ShareAlike 4.0 International License which allows others to remix, tweak, and build upon the work non-commercially, as long as the author is credited and the new creations are licensed under the identical terms.
}

dosage forms; it will improve bioavailability, enhancement of therapeutic efficacy, avoid limitations of the first-pass effect, and maintenance of steady plasma level of medicine. $[2,3]$ There are mainly two types of TDS matrix type and reservoir type TDS. Matrix type TDS contains drugs in pressure-sensitive adhesion, and the reservoir type system may contain drugs in solution or PSA, but there is the rate-controlling membrane to manage the delivery of medicine ${ }^{[4]} \mathrm{RM}$ is used to treat early Parkinson's disease, and it is an irreversible inhibitor of monoamine oxidase 
type B (MAO-B). ${ }^{[5]} \mathrm{RM}$ is selected as a model drug for TDS development as the drug has low bioavailability (36\%) and undergoes extensive first-pass metabolism. RM has all the ideal properties to develop a TDS like low dose (1 mg/day), shorter half-life (3 hours), molecular weight (267.35 g/mol), and partition coefficient Log $P(2.24) .{ }^{[6]}$ A basic component of the TDS is a backing film to support the adhesive matrix, PSAs as a matrix to control the release and provide adhesion properties to patch, the rate-controlling membrane and release liner to protect the patch during storage. ${ }^{[7,8]}$ In a present research study, the industrials approach was adopted for the development of a TDS. Various preformulation studies like solubility of a drug in various solvents and permeation enhancers, transmission and uptake study, drug-excipient compatibility study and crystallization study in different type PSA were performed. Selection and optimization of PSA and permeation enhancers were done based on in-vitro adhesion testing and penetration study on human cadaver skin by modified Franz diffusion cell system. A total of thirteen different formulations were formulated to select and optimization of PSA, a permeation enhancer, and dry GSM of a matrix. The final transdermal patches were tested for appearance, the weight of matrix, thickness, percentage assay of drug content, in-vitro adhesion testing, cold flow study, ex-vivo skin permeation studies, and stability study.

\section{Materials AND METHODS}

\section{Materials}

The RM was obtained free of charge from Apotex Research Pvt. Ltd., Bangalore, India. PSA Durotak 87-4098, Durotak 87-2051, and Durotak 87-2287 were obtained as a gift sample from Henkel (Drogenbos, Belgium). Human cadaver skin samples were obtained from Science Care Inc. (Philadelphia, USA). Propylene glycol, Lauryl lactate and isopropyl palmitate were supplied by Finar Chemical Corporation Pvt. Ltd. Ahmedabad, India. Oleic acid was obtained from the
Croda Chemicals (India) Pvt. Ltd. Mumbai, India). Scotchpak 9723, Cotran 9720, Scotchpak 9757, and Scotchpak 1109 were obtained from 3M India Ltd. Mumbai, India. Silicon coated polyester film was received as gift samples from Saint Gobain. PET film XL was supplied by Kaygee- Loparex India Pvt. Ltd. Gujarat, India. Other analytical grade chemicals, solvents, and reagents were used.

\section{Fabrication of Rasagiline Mesylate Drug in Adhesive Patches}

Precisely weighed RM was dissolved in ethyl acetate under mixing. A permeation enhancer was added and mixed it for 10 minutes. The PSA was added slowly under mixing. The blend was mixed for 10-15 minutes for uniform mixing of all ingredients. A blend was coated on silicone coated surface of release liner with uniform thickness to achieve 150 dry GSM in Mathis-I Lab coater for 20 minutes at $80^{\circ} \mathrm{C}$. Dried sheets were laminated with backing film using Benchtop laminator. Prepared laminates were die cut using $15 \mathrm{~cm}^{2}$ die. Die-cut patches were pouched in paper pouches and stored at controlled room temperature till further evaluation (Table 1).

\section{Evaluation of Rasagiline Mesylate Transdermal Patches}

\section{Preformulation Studies}

Solubility studies in different permeation enhancers were carried out by preparing a saturated solution of drug in different permeation enhancers and solvents. In the drugexcipient compatibility study, the drug was mixed with various excipients (PSA, permeation enhancer) in 1:1 ratio. This mixture was kept in glass vials than properly capped and sealed with teflon tape. Two vials of each mixture were put at controlled room temperature $\left(25^{\circ} \mathrm{C}\right)$ and in the hot air oven at $40^{\circ} \mathrm{C}$ for one month period. A transmission study was done in order to check the permeability of the drug through backing film and liner. This study is critical

Table 1: Formulation composition of rasagiline mesylate transdermal system

\begin{tabular}{lllll}
\hline Formulation code & Rasagiline mesylate $(\% w / w)$ & Pressure-sensitive adhesive $(\% w / w)$ & Permeation enhancer $(\% w / w)$ & Dry $G S M$ \\
\hline FD-1 & $2 \%$ & $98 \%$ DT-6908 & - & 150 \\
FD-2 & $5 \%$ & $95 \%$ Bio PSA 4202 & - & 150 \\
FD-3 & $14 \%$ & $86 \%$ DT-4098 & $3 \%$ (Propylene glycol) \\
FD-4 & $14 \%$ & $83 \%$ DT-4098 & $3 \%$ (Lauryl lactate) \\
FD-5 & $14 \%$ & $83 \%$ DT-4098 & $3 \%$ (Isopropyl palmitate) & 150 \\
FD-6 & $14 \%$ & $83 \%$ DT-4098 & $3 \%$ (Oleic acid) & 150 \\
FD-7 & $14 \%$ & $83 \%$ DT-4098 & $3 \%$ (Lauryl lactate) \\
FD-8 & $14 \%$ & $83 \%$ DT-4908 & $5 \%$ (Lauryl lactate) \\
FD-9 & $14 \%$ & $81 \%$ DT-4908 & $7 \%$ (Lauryl lactate) \\
FD-10 & $14 \%$ & $79 \%$ DT-4908 & $5 \%$ (Lauryl lactate) \\
FD-11 & $14 \%$ & $81 \%$ DT-4908 & $5 \%$ (Lauryl lactate) \\
FD-12 & $14 \%$ & $81 \%$ DT- 4908 & $5 \%$ (Lauryl lactate) \\
FD-13 & $14 \%$ & $81 \%$ DT- 4908 & & 150 \\
\hline
\end{tabular}


in the selection of the backing and liner. If the drug is permeable through any of these, it may cause formulation error, and drug content in a final formulation may decrease as some drug may leach or transmit through backing or liner during the formulation. Uptake study was done in order to check the percent drug uptake or absorbed by backing and liner. A saturated solution of RM was prepared in lauryl lactate. Several pieces of backing film/ release liners were cut of $12.56 \mathrm{~cm}^{2}$ size and applied on Franz diffusion cell. The donor cell was then mounted above it, and the two cells were clamped tightly so that the backing film/release liners mounted on the receptor was sandwiched between the donor and the receptor cells. The receptor cell of the diffusion cell apparatus was filled with phosphate-buffered saline $\mathrm{pH}$ 7.4.2 mL of a saturated solution of RM was filled in the donor compartment using a syringe. A magnetic stirrer was used to keep the receptor media under stirring. The temperature was maintained at $40^{\circ} \mathrm{C}$. The receptor phase was sampled at initial, 24,48 , 96,120 , and 168 hours, and the withdrawn samples were analyzed for RM content. For uptake study, a saturated solution of RM was prepared in lauryl lactate. Several $1 \mathrm{~cm}^{2}$ pieces of backing films/release liners were cut and immersed in RM-lauryl lactate solution and stored at $40^{\circ} \mathrm{C} / 75 \% \mathrm{RH}$ and $25^{\circ} \mathrm{C} / 60 \% \mathrm{RH}$. The backing film/ release liner was sampled at the interval of 2,4 , and 6 weeks. The sample was wiped carefully and analyzed for RM content. For a crystallization study, patches were prepared with different concentrations of API and stored at different stability conditions as follows: Freeze-thaw cycle accelerated stability, 90\% RH and controlled room temperature for 60 days.

The final transdermal patches were evaluated for appearance, weight of matrix, thickness, \% assay of drug, in-vitro adhesion testing, cold flow study, and ex-vivo skin permeation studies.

\section{Appearance}

Prepared TDS were inspected for appearance visually.

\section{Weight of Matrix}

The total weight of each patch was taken individually, and the weight of matrix calculate by removing tare weight of release liner and backing film and mean value calculated. ${ }^{[9,10]}$

\section{Thickness of Patch}

The thickness of patches was measured using micrometer at five different places of patch and mean values were calculated. ${ }^{[11]}$

\section{Rasagiline Mesylate (RM) Content by High- Performance Liquid Chromatography (HPLC)}

The RM content was determined by developed high performance liquid chromatographic method. ${ }^{[12,13]}$
Methanol and phosphate buffer ( $\mathrm{pH}$ 3.0) was used as mobile phase. Following chromatography, the condition was used for the analysis of RM content in the TDS.

$\begin{array}{lcl}\text { Column } & : & \text { Prontosil C18 } \\ \text { Flow rate } & : & 1.0 \mathrm{~mL} / \mathrm{min} \\ \text { Wavelength } & : & 210 \mathrm{~nm} \\ \text { Injection volume } & : & 50 \mu \mathrm{L} \\ \text { Column temperature: } & 35^{\circ} \mathrm{C} \\ \text { Sample temperature }: & 25^{\circ} \mathrm{C} \\ \text { Mode } & : & \text { Gradient } \\ \text { Run time } & : & 12 \mathrm{~min}\end{array}$

\section{In-vitro Adhesion Testing (Peel, Tack, and Shear)}

In-vitro adhesion properties of patch are characterized by peel strength (minimal force which takes to remove the patch from its surface), shear strength (the patch applies a resistances against the flow/detachment) and tack strength (the property of the patch to form bond with the contact surface under light pressure and brief contact). ${ }^{[14]}$ Tack is the early physical bonding of patch onto the skin normally occurs in a few seconds. Tack was measured using Lloyd (AMETEX) instrument. One patch was taken and cut it to a size of 1 -inch square. Remove release liner was removed and applied on test panel such way that adhesion side remain upward direction towards the hole. A machine was started at a speed of $610 \mathrm{~mm} / \mathrm{min}$ to bring a contact of the probe to the adhesive site of patch. After 1 second contact time, removed probe from adhesive at the same speed. Note downforce (maximum) required for removing the probe from patches. Shear was measured using Chem. Instrument (10 bank shear tester). The patch was cut of 0.5 -inch width. A patch was adhered on to the stainless steel plate up to 0.5 -inch mark portion of the release liner. Roll the roller on it and allow it to stand for 15 minutes. The other end was attached with a hook, and after completion of required dwell time, $100 \mathrm{gm}$ weight was hanged on the hook. The time required to fall down the patch was measured.

\section{Cold Flow Study of Rasagiline Mesylate Patch}

Cold flow is the movement of adhesive outside the borders of a TDS during storage. The cold flow was measured by a microscopical method by viewing the patch under a microscope at initial and after 1-month when stored at $40^{\circ} \mathrm{C} / 75 \% \mathrm{RH}^{[15-17]}$

\section{Ex-vivo Permeation Study of Rasagiline Mesylate Patch}

Hairless human cadaver skin is used for permeation studies. Dermatomed of human cadaver skin was received from the vendor. Stratum corneum of skin was separated from dermatomed skin by putting the skin hot water for 2-3 hours at $55 \pm 5^{\circ} \mathrm{C}$ using a cotton swab. Separated stratum corneum was dried overnight at controlled 
room temperature. After drying of the skin, it was stored at $2-8^{\circ} \mathrm{C}$ till further used. The Ex-vivo permeation study was performed using a modified Franz diffusion cell. The excised human cadaver skin was cut into a size of $2.0 \mathrm{~cm}^{2}$ using die cutter and placed between the receptor and donor compartments so that the dermal side of the skin faced the receiver fluid. The release liner was removed from the patch and applied to the skin. The specified volume of diffusion medium was filled into the Franz diffusion cell. Franz diffusion cell was placed on a magnetic stirrer, and small magnetic bead was added and stirred the content under 500 RPM to keep them well mixed. Aliquots of a diffusion medium were removed at specified sampling points and filtered through Whatman $\AA$ filter grade 41 . An amount of drug was determined by HPLC using an appropriate method, as discussed earlier. The same volume of fresh diffusion medium after each removal was reinstated into the diffusion cell to maintain sink condition. The study was continued up to 168 hours. Each study was carried out on three skin $(n=3)$, and mean flux was calculated. ${ }^{[18-21]}$

\section{Stability Study}

The optimized formulation (FD13) of the RM TDS were pouched in paper pouch (41GSM Paper/17 GSM LDPE/9 $\mu$ $\mathrm{Al} / 35 \mathrm{GSM}$ LDPE) and kept at $25^{\circ} \mathrm{C} / 65 \%$ R.H. and $40^{\circ} \mathrm{C} / 75 \%$ R.H. for 3 months. Various stability tests like appearance, assay, related substances, in-vitro adhesion, and cold flow of patches were performed at different time points.

\section{RESULTS}

RM patches were prepared using solved casting method. First of all, preformulation studies were carried out to select backing films, release liner, a PSA, drug loading capacity in various PSAs. Solubility RM was found 55.42 $\mathrm{mg} / \mathrm{mL}, 29.2 \mathrm{mg} / \mathrm{mL}, 12.8 \mathrm{mg} / \mathrm{mL}$ and $6.59 \mathrm{mg} / \mathrm{mL}$ in lauryl lactate, oleic acid isopropyl palmitate and propylene glycol respectively. RM is freely soluble in ethanol and ethyl acetate. Drug excipient compatibility study did not show any significant change in the assay and related substances, so RM is compatible with various PSAs (DT-6908, DT-4098, and Bio PSA-4202) and permeation enhancers. For the crystallization study, various patches were prepared with different drug concentrations in different PSAs. There was no crystal found in DT-4098 up to $14 \%$ drug concentration, In Bio PSA 4202 up to $5 \%$ drug concentration and in DT-6908 up to 3\% drug concentration. Patches were prepared with 16\% drug concentration in DT-4098 showing crystals as shown in Fig. 1. Backing film Scotchpak 9723 and release liner SG-8310 was not showing any transmission and uptake of RM.

\section{Evaluation of Rasagiline Mesylate Transdermal Patches}

\section{Appearance}

All patches of different formulation were rectangular patch with round edges comprising rectangular transparent release liner with beige color backing. Upon removal of the transparent release liner, a uniform matrix layer on the backing is visible, substantially free of external particulate matter and bubbles.

\section{Weight of Matrix Uniformity}

The weight of matrix of all formulations is uniform. The values of all formulations vary from $121.6 \pm 2.4 \mathrm{mg}$ (80 GSM) to $227.0 \pm 1.1 \mathrm{mg}$ (150 GSM) (Tables 2 and 3 ).

\section{Thickness of Patch}

The thickness of patches ensured uniformity of thickness in all developed formulation. The value ranges from $241.2 \pm$ $1.3 \mu \mathrm{m}$ (80 GSM) to $264 \pm 1.4 \mu \mathrm{m}$ (150 GSM) (Tables 2 and 3).

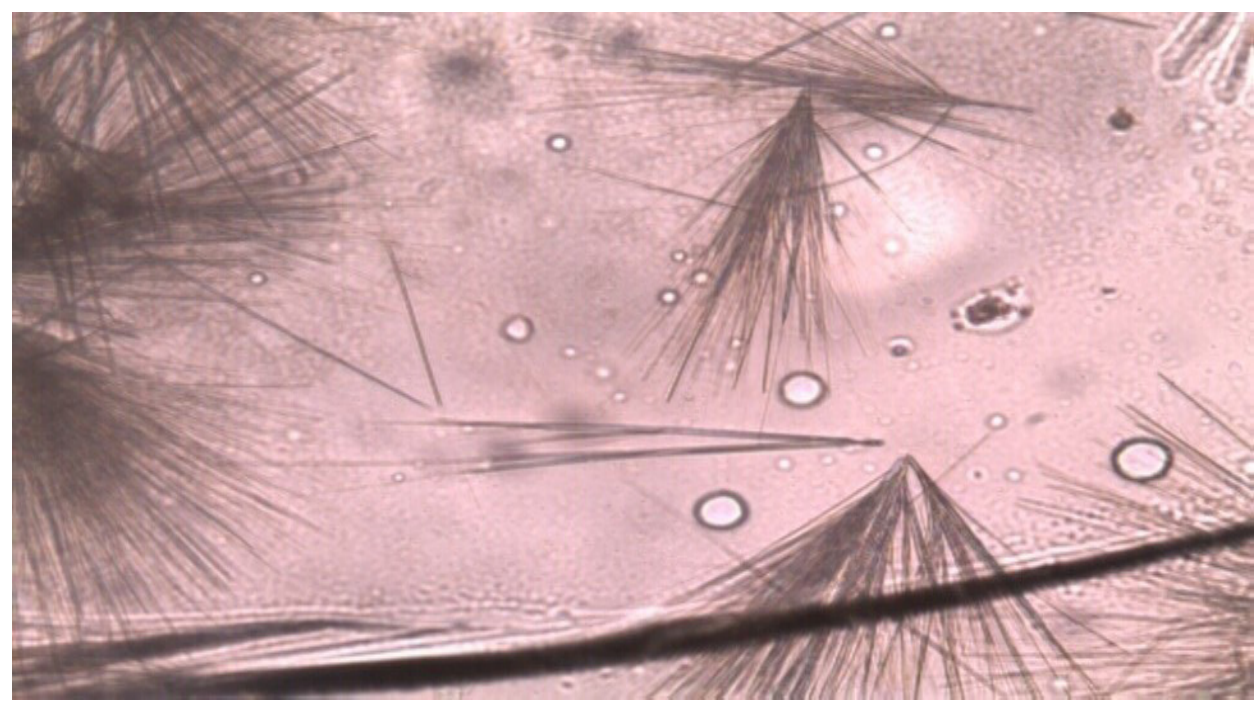

Fig. 1: Patches with crystal of rasagiline mesylate 
Design, Development, and Delivery of Rasagiline Mesylate from Monolithic Drug in Adhesive Matrix Patches

Table 2: Results of evaluation of rasagiline mesylate patch

\begin{tabular}{|c|c|c|c|c|c|c|c|}
\hline \multirow[b]{2}{*}{ Tests } & \multicolumn{3}{|c|}{ Selection of pressure-sensitive adhesive types } & \multicolumn{4}{|c|}{ Selection of Permeation enhancer } \\
\hline & $F D-1$ & $F D-2$ & $F D-3$ & $F D-4$ & $F D-5$ & $F D-6$ & $F D-7$ \\
\hline Matrix weight, mg & $226 \pm 1.6$ & $225.4 \pm 1.9$ & $227 \pm 1.1$ & $224.2 \pm 1.6$ & $223.8 \pm 2.4$ & $224.6 \pm 3.1$ & $225 \pm 1.8$ \\
\hline Patch thickness, $\mu \mathrm{m}$ & $263.6 \pm 1.8$ & $263.4 \pm 1.3$ & $263 \pm 2.3$ & $261.6 \pm 1.2$ & $261.4 \pm 2.7$ & $261 \pm 3.0$ & $261.4 \pm 3.4$ \\
\hline $\begin{array}{l}\text { Drug content \% label } \\
\text { claim }\end{array}$ & $99.26 \pm 0.7$ & $99.38 \pm 0.6$ & $103.6 \pm 1.1$ & $97.9 \pm 1.4$ & $100.8 \pm 3.1$ & $99.44 \pm 2.9$ & $102.2 \pm 1.9$ \\
\hline Peel, gm force & $4.6 \pm 1.1$ & $5.4 \pm 0.9$ & $13.6 \pm 1.1$ & $13.6 \pm 1.1$ & $14.4 \pm 1.5$ & $13.4 \pm 1.8$ & $13.6 \pm 1.5$ \\
\hline Tack, gm $/ \mathrm{mm}^{2}$ & $10.4 \pm 0.9$ & $31.2 \pm 4.4$ & $10.8 \pm 0.8$ & $13.2 \pm 1.6$ & $13.6 \pm 2.1$ & $14.2 \pm 1.9$ & $13.2 \pm 1.6$ \\
\hline Shear adhesion, minutes & $114.8 \pm 9.1$ & $14.2 \pm 3.4$ & $76 \pm 4.2$ & $76 \pm 4.2$ & $81 \pm 9.6$ & $79 \pm 6.5$ & $83 \pm 1.6$ \\
\hline Release force $\mathrm{g} / \mathrm{cm}$ & $7.4 \pm 1.8$ & $8.8 \pm 0.4$ & $7.6 \pm 1.1$ & $7.8 \pm 1.3$ & $8 \pm 1.2$ & $7.8 \pm 1.3$ & $7.8 \pm 1.3$ \\
\hline Cold flow (\%) & $2.1 \pm 0.3$ & $10.1 \pm 0.4$ & $3.5 \pm 0.2$ & $3.1 \pm 0.4$ & $3.5 \pm 0.3$ & $3.4 \pm 0.4$ & $3.2 \pm 0.2$ \\
\hline $\begin{array}{l}\text { Average cumulative } \\
\text { permeation, } \mu \mathrm{g} / \mathrm{cm}^{2}\end{array}$ & $111.33 \pm 7.23$ & $124.67 \pm 3.79$ & $188.33 \pm 4.93$ & $206 \pm 2.65$ & $287.33 \pm 13.43$ & $219 \pm 1.0$ & $256 \pm 11.27$ \\
\hline Flux $\left(\mu \mathrm{g} / \mathrm{cm}^{2} / \mathrm{hr}\right)$ & $0.67 \pm 0.10$ & $0.79 \pm 0.10$ & $1.23 \pm 0.09$ & $1.33 \pm 0.08$ & $1.82 \pm 0.20$ & $1.44 \pm 0.14$ & $1.65 \pm 0.18$ \\
\hline
\end{tabular}

Table 3: Results of evaluation of rasagiline mesylate patch

\begin{tabular}{|c|c|c|c|c|c|c|}
\hline \multirow[b]{2}{*}{ Tests } & \multicolumn{3}{|c|}{ Optimization of concentration of permeation enhancer } & \multicolumn{3}{|c|}{ Matrix thickness or dry GSM versus drug delivery } \\
\hline & $F D-8$ & $F D-9$ & $F D-10$ & $F D-11$ & $F D-12$ & $F D-13$ \\
\hline Matrix weight, mg & $224.8 \pm 2.0$ & $225 \pm 1.6$ & $224.2 \pm 1.6$ & $121.6 \pm 2.4$ & $151.4 \pm 2.6$ & $225.6 \pm 2.8$ \\
\hline Patch thickness, $\mu$ & $264 \pm 1.4$ & $262.6 \pm 1.8$ & $260.4 \pm 1.9$ & $241.2 \pm 1.3$ & $252.2 \pm 1.6$ & $262.6 \pm 1.8$ \\
\hline Drug content \% Label claim & $100.3 \pm 2.7$ & $100.6 \pm 3.0$ & $99.4 \pm 2.7$ & $99.92 \pm 3.1$ & $101.4 \pm 2.6$ & $98.4 \pm 2.8$ \\
\hline Peel, gm force & $14.2 \pm 1.5$ & $24.2 \pm 2.4$ & $24.2 \pm 2.4$ & $5.4 \pm 1.1$ & $18.8 \pm 0.8$ & $25.8 \pm 2.8$ \\
\hline Tack, gm/19.6 mm² & $13.6 \pm 2.1$ & $16.6 \pm 1.1$ & $16.8 \pm 1.3$ & $8.6 \pm 1.5$ & $11.2 \pm 1.1$ & $17.2 \pm 1.2$ \\
\hline Shear adhesion, minutes & $82 \pm 8.4$ & $63.2 \pm 3.3$ & $64.2 \pm 2.8$ & $127 \pm 2.7$ & $89.8 \pm 6.1$ & $64.4 \pm 4.0$ \\
\hline Release force & $8 \pm 1.2$ & $8 \pm 0.7$ & $8 \pm 0.7$ & $7.6 \pm 1.1$ & $7.8 \pm 1.3$ & $8 \pm 0.7$ \\
\hline Cold flow (\%) & $2.9 \pm 0.3$ & $3.5 \pm 0.4$ & $4.5 \pm 0.3$ & $1.5 \pm 0.2$ & $2.5 \pm 0.4$ & $3.9 \pm 0.1$ \\
\hline $\begin{array}{l}\text { Average cumulative } \\
\text { permeation, } \mu \mathrm{g} / \mathrm{cm}^{2}\end{array}$ & $287.33 \pm 13.43$ & $378 \pm 1.0$ & $443 \pm 2.65$ & $180.33 \pm 4.93$ & $205.33 \pm 1.53$ & $378 \pm 1.0$ \\
\hline Flux $\left(\mu \mathrm{g} / \mathrm{cm}^{2} / \mathrm{hr}\right)$ & $1.82 \pm 0.20$ & $2.36 \pm 0.07$ & $3.01 \pm 0.06$ & $1.27 \pm 0.08$ & $1.44 \pm 0.13$ & $2.36 \pm 0.07$ \\
\hline
\end{tabular}

Rasagiline Mesylate Content by High-Performance Liquid Chromatography

The value ranges from $97.9 \pm 1.4 \%$ to $103.6 \pm 1.1$ (Tables 2,3 ).

From the thickness of matrix, weight of matrix and drug content, it was confirmed that uniformity of all formulation giving reproducible results by the proposed manufacturing method.

\section{In-vitro Adhesion testing (Peel, Tack, and Shear)}

Effect of PSAs on adhesion properties: The data of Table 2 shows that formulation FD-3, which having Durotak 87-4098, shows higher peel value compare to formulations fatal dose-1 (FD-1) and fatal dose-2 (FD-2) having Durotak 6908 and Bio PSA-4202, respectively. Thus, as acrylate polymer is suitable for long wear TDS. Formulation FD2 showed higher tack but low shear while formulation FD1 showed low peel, tack, and higher shear.

Effect of permeation enhancer on adhesion properties: The data of Table 3 shows increase in the permeation enhancer, adhesive force increases (peel and tack value) and cohesive force (shear) value decreases FD8 and FD9. This indicates that the permeation enhancer has a plasticizer effect due to the alteration of Tg value of the PSA of the system. ${ }^{[22]}$

Effect of dry GSM on adhesion properties: The data of Table 3 shows that as GSM of matrix increases, a continuous increase in the peel and tack was naturally associated with a decrease in shear adhesion.

\section{Cold Flow Study of Rasagiline Mesylate Patch}

From Tables 2 and 3, as we increase the tack of a patch, there was an increase in the cold flow of patch. Also as we increase the dry GSM of patch, there was increase in the cold flow. Patches having high tack and low shear value showed higher cold flow.

\section{Ex-vivo Permeation Study of Rasagiline Mesylate Patch}

Ex-vivo permeation study of formulated transdermal patches was performed through human cadaver skin by Franz diffusion cell. The maximum thermodynamic activity can be achieved when the highest drug concentration in the matrix of patches. The average cumulative amount of RM that permeated across the skin, over 168 hours, from FD 1- 2\% API, FD 2-5\% API, FD 3-14\% API was found to be 
$111.33 \pm 7.23 \mu \mathrm{g} / \mathrm{cm}^{2}, 124.67 \pm 3.79 \mu \mathrm{g} / \mathrm{cm}^{2}$ and $188.33 \pm$ $4.93 \mu \mathrm{g} / \mathrm{cm}^{2}$ respectively. Batch FD-3 showed significantly higher cumulative drug delivery compared to the other two batches, as presented in Fig. 2. The average permeation rate of FD-1, FD-2 and FD-3 was found to be $0.67 \pm 0.10$ $\mu \mathrm{g} / \mathrm{cm}^{2} / \mathrm{hr}, 0.79 \pm 0.10 \mu \mathrm{g} / \mathrm{cm}^{2} / \mathrm{hr}$ and $1.23 \pm 0.09 \mu \mathrm{g} /$ $\mathrm{cm}^{2} / \mathrm{hr}$ respectively, where the highest permeation rate was obtained from FD-3, as presented in Fig. 3. The high permeation rate of RM from batch FD-3 can be imputed to the high drug concentration $(14 \% \mathrm{w} / \mathrm{w})$.

From Tables 2 and 3, the penetration rate of RM in the existence of different permeation enhancers intended for enhancing the drug permeation. The RM permeation was significantly enhanced with the addition of permeation enhancers. Among the penetration enhancers evaluated, the permeation rate was decreased as; lauryl lactate $>$ oleic acid > isopropyl palmitate> Propylene glycol formulation FD3 (without any permeation enhancers). The total quantity of RM transported in $168 \mathrm{~h}$ was 287.33 $\pm 13.43 \mu \mathrm{g} / \mathrm{cm}^{2}, 256.00 \pm 11.27 \mu \mathrm{g} / \mathrm{cm}^{2}, 219.00 \pm 1.00 \mu \mathrm{g} /$ $\mathrm{cm}^{2}$ and $206.0 \pm 2.65 \mu \mathrm{g} / \mathrm{cm}^{2}$ when treated with lauryl lactate, oleic acid, isopropyl palmitate, and propylene glycol, respectively.

The greatest skin flux of RM observed $\left(2.36 \mu \mathrm{g} / \mathrm{cm}^{2} / \mathrm{h}\right.$ at 12 and 24 hours) when formulation contain lauryl lactate among various formulations evaluated in this study, under similar experimental conditions. In addition, Lauryl lactate shows higher flux during 168 hours period when compared with the rest three permeation enhancers.

Formulation FD-8, FD-9, and FD-10 were prepared with 3, 5, and 7\% lauryl lactate for permeation study. From Fig. 4, it is clear that as an increase in lauryl lactate concentration increases the cumulative penetration and permeation rate of RM.

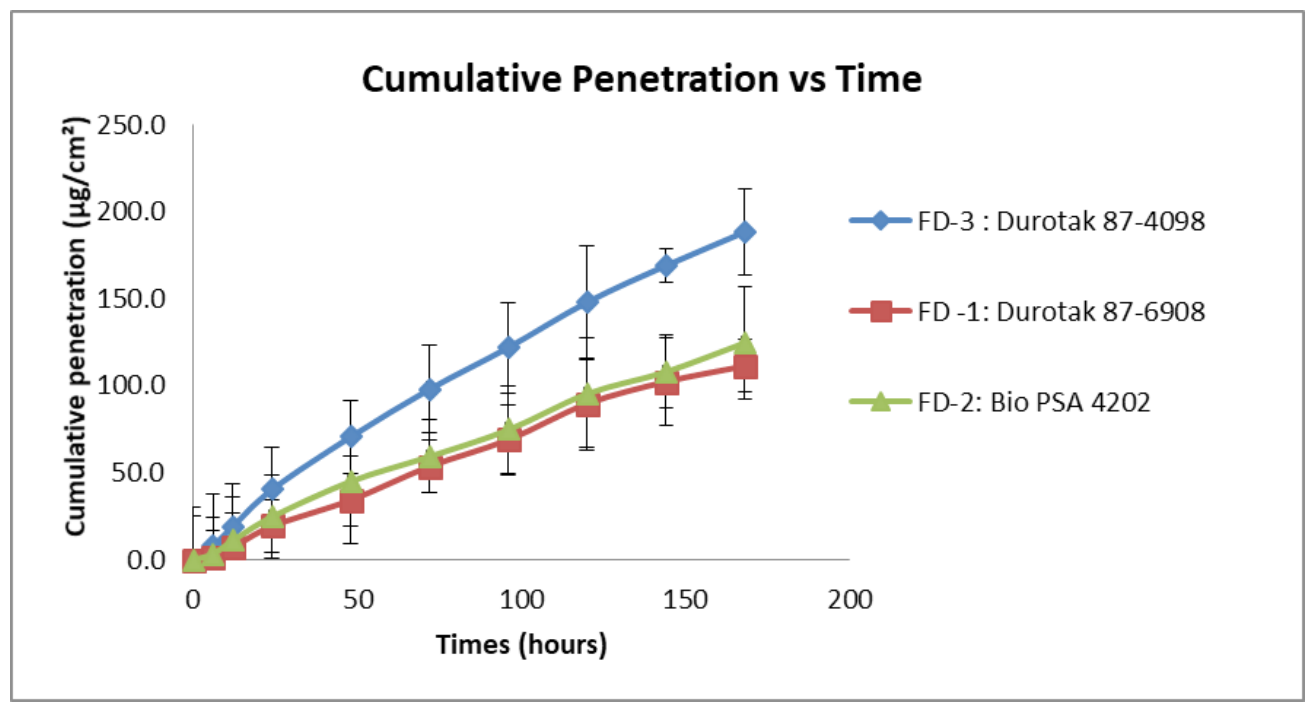

Fig. 2: Effect of pressure sensitive on cumulative permeation (FD1, FD2, and FD3)

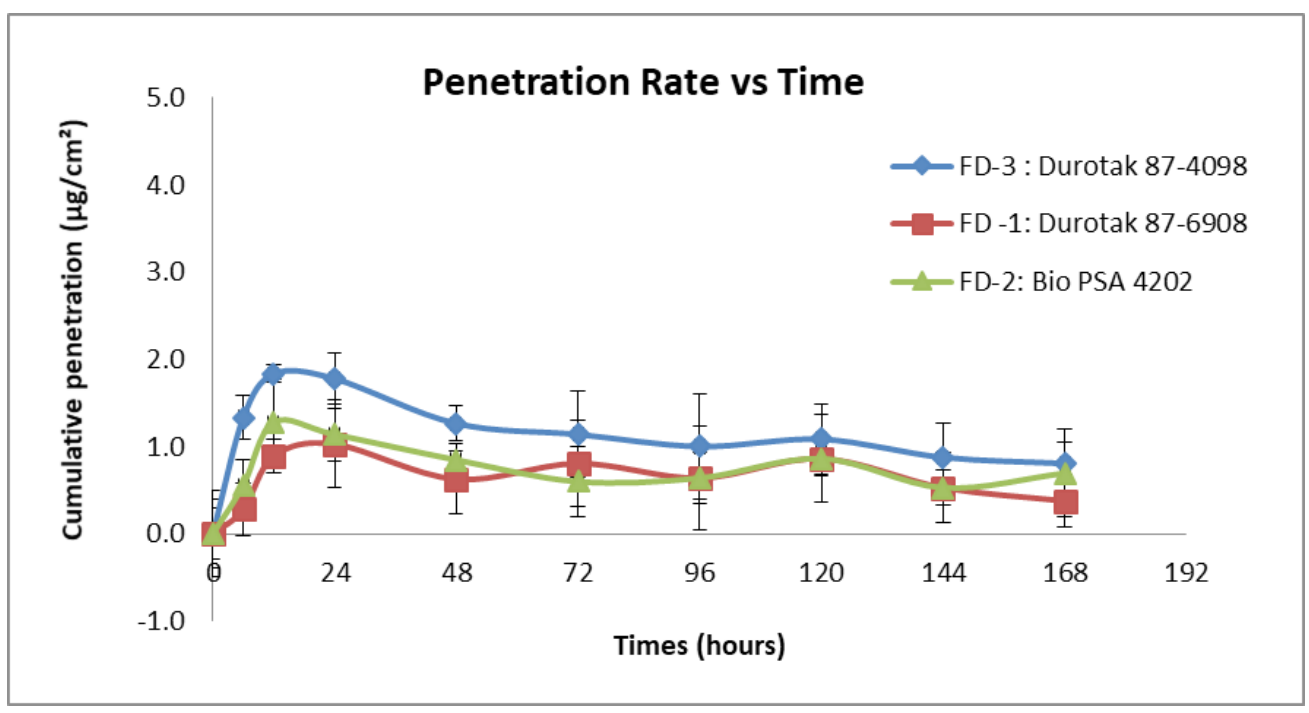

Fig. 3: Effect of pressure sensitive adhesive on the rate of permeation (flux rate) 


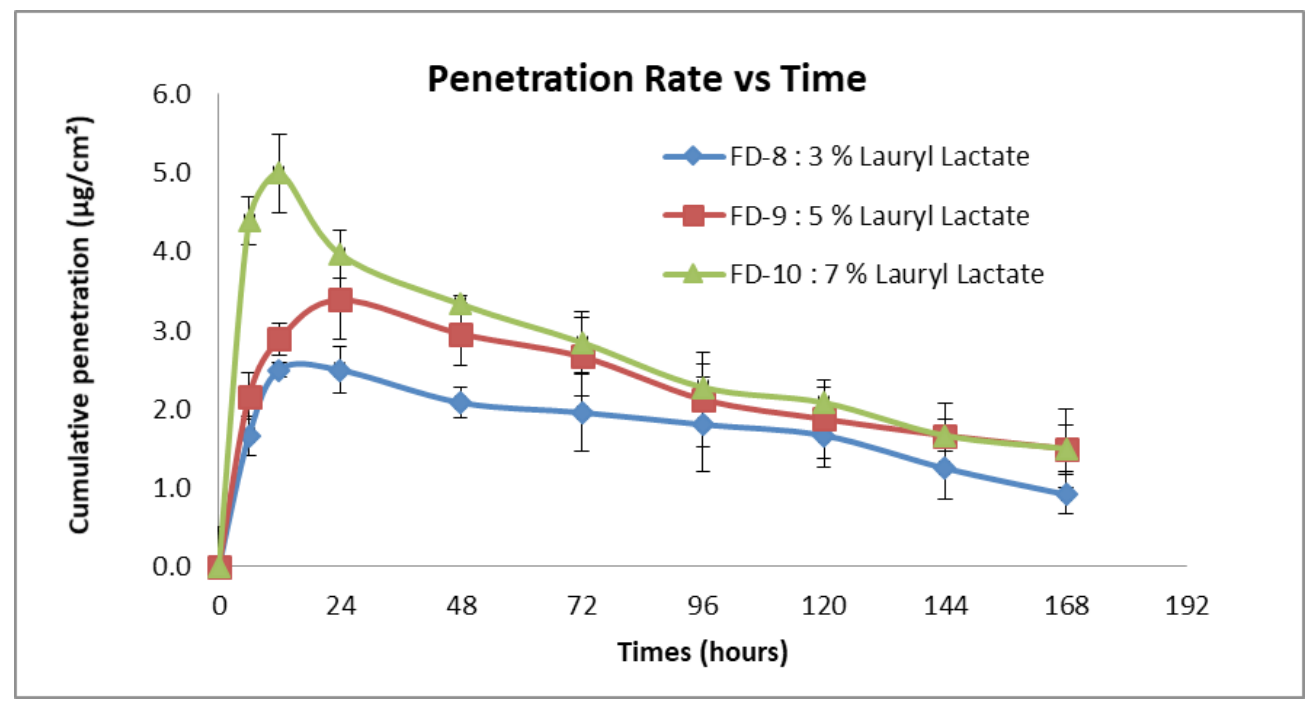

Fig. 4: Effect of different concentration of lauryl lactate on the penetration rate of rasagiline mesylate

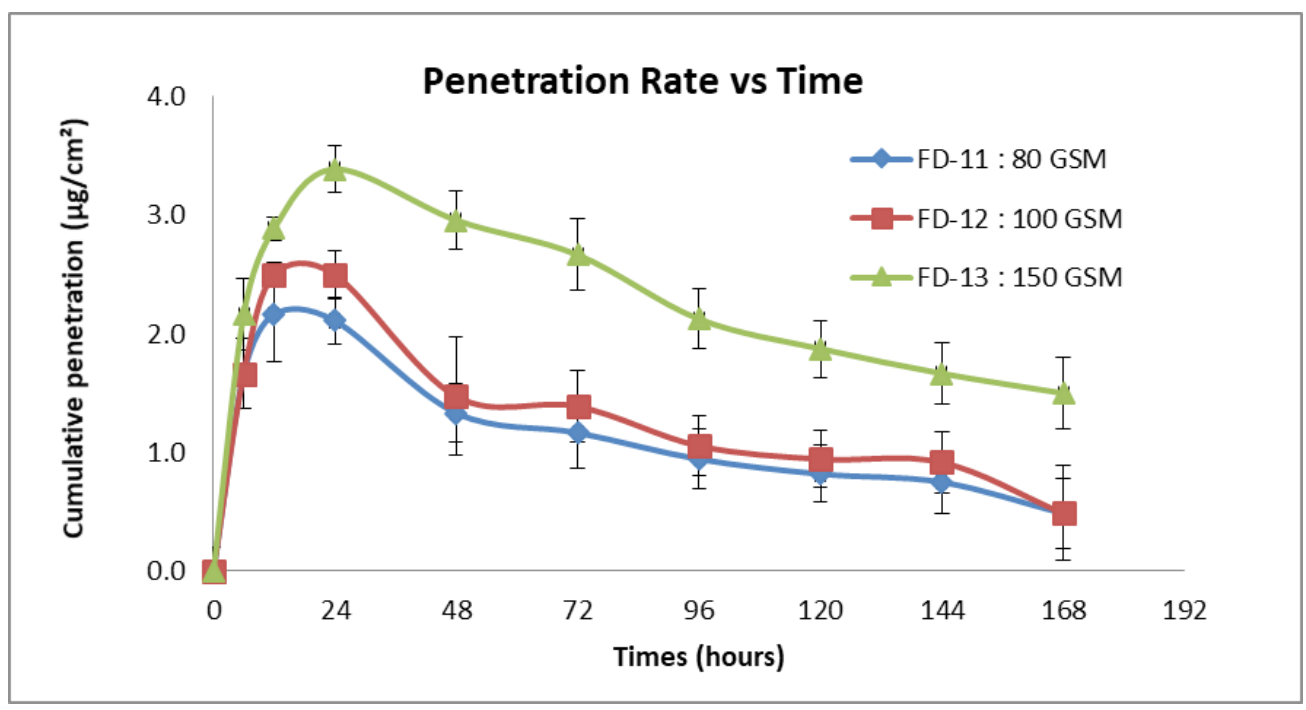

Fig. 5: Effect of dry GSM on cumulative penetration of rasagiline mesylate

The average cumulative amount of RM that permeated through the skin after 168 hours, from FD11-80 GSM, FD12-100 GSM, FD13-150 GSM were found to be 180.33 $\pm 4.99 \mu \mathrm{g} / \mathrm{cm}^{2}, 205.33 \pm 1.53 \mu \mathrm{g} / \mathrm{cm}^{2}$ and $378.00- \pm 1.00$ $\mu \mathrm{g} / \mathrm{cm}^{2}$ respectively. Formulation FD13 showed drastically higher cumulative drug delivery compared to the other two formulations. The average flux of FD11, FD12 and FD13 was found to be $1.27 \pm 0.08 \mu \mathrm{g} / \mathrm{cm}^{2} / \mathrm{hr}, 1.44 \pm 0.13 \mu \mathrm{g} /$ $\mathrm{cm}^{2} / \mathrm{hr}$ and $2.36 \pm 0.07 \mu \mathrm{g} / \mathrm{cm}^{2} / \mathrm{hr}$ respectively, where the highest flux was obtained from FD13, as presented in Fig. 5. Data shows that as dry GSM (Matrix thickness) increases in a formulation, cumulative amount permeated, and the rate of drug permeation is increased. There is a slight increase in flux for formulation having 100 GSM compares to 80 GSM, but significantly increases in a formulation having 150 GSM. In FD13 formulations, the rate of permeation maintains up to 96 hours and then starts decreasing after
96 hours. While for FD11 and FD12 formulations, the rate of permeation is higher up to 12 and 24 hours, respectively, compared to FD13 formulation and decrease to a similar level of FD13 at 12 and 24 hours, respectively. These data indicate that an increase in GSM of the TDS increases the delivery rate at later time points, and it is mostly due to the constant concentration gradient for the entire time.

\section{Stability study}

Stability study of formulation FD13 was performed at $25^{\circ} \mathrm{C} / 65 \%$ R.H. and $40^{\circ} \mathrm{C} / 75 \%$ R.H storage conditions up to 3 months. As per the stability plan, the patches were withdrawn and tested for appearance, \% assay of drug content, related substances, in-vitro adhesion, and cold flow. Stability results at CRT and accelerated storage condition were found to be satisfactory for up to three months. 
Vipulbhai Mandli et al.

\section{DISCUSSION}

A systematic development was followed to develop drug in adhesive rasagiline mesylate transdermal system (ARMTS) using various parameters to developed an effective delivery system. The effect of various formulation factors like PSAs, skin permeation enhancers, and solubility were examined. Based on results, the development of the RM TDS was successful with Durotak 87-4098 PSA, which can provide sustain drug delivery up to 168 hour period. Based on the crystallization study, RM has a higher solubility in acrylate adhesive compare to polyisobutylene adhesive and silicone adhesive. The drug has around 14\% w/w solubility in Durotak 87-4098. Release liner SG-8310 and backing film Scotchpak 9723 was selected based on uptake and transmission study. Different permeation enhancers were evaluated and formulation with lauryl lactate has found higher flux compare to other permeation enhancers like oleic acid, propylene glycol, isopropyl palmitate, and propylene glycol. Formulation with 5\% lauryl lactate shows appropriate flux and adhesion properties like peel, tack, and shear for a 7-day wear period. Matrix thickness also plays an important role in the determination of in vitro adhesion characteristics; as matrix thickness (GSM) increases, a continuous increase in the peel was naturally associated with the reduction in shear adhesion. Ex-vivo permeation study evident that the level of enhancer and GSM of the formulation is directly proportional to the cumulative drug amount and skin flux rate. Formulation with Durotak 87-4098 having 14\% drug concentration and 5\% lauryl lactate as penetration enhancer showing good permeation rate $\left(2.36 \pm 0.07 \mu \mathrm{g} / \mathrm{cm}^{2} / \mathrm{hr}\right)$, appropriate adhesion properties (Peel, tack and shear) for a 7-day wear period, low cold flow and good stability data up to 3 months at $25^{\circ} \mathrm{C} / 60 \% \mathrm{RH}$ and $40^{\circ} \mathrm{C} / 75 \% \mathrm{RH}$.

\section{REFERENCES}

1. Guy RH, Hadgraft J. Transdermal Drug Delivery. Edn 2, Marcel Dekker Inc, New York. 2003;371-377.

2. Keith AD. Polymer matrix considerations for transdermal devices. Drug development and industrial pharmacy. 1983 Jan 1; 9(4): 605625.

3. Tanwar H, Sachdeva R. Transdermal drug delivery system: A review. International Journal of Pharmaceutical Sciences and Research. 2016;7:2274-2290.

4. Dipen Patel, Sunita A. Chaudhary, Bhavesh Parmar, Nikunj Bhura. Transdermal drug delivery system: a review. The pharma innovation. 2012;1:66-75.

5. Vicki Oldfield, Gillian M. Keating, Caroline M. Perry. Rasagiline: a review of its use in the management of Parkinson's disease. Drugs. 2007;67:1725-1747.

6. Chandrashekar NS, Shobha Rani RH. Physicochemical and pharmacokinetic parameters in drug selection and loading for transdermal drug delivery. Indian journal of pharmaceutical sciences. 2008;70:94-96.

7. Gaikwad AK. Transdermal drug delivery system: Formulation aspects and evaluation. Comprehensive Journal of Pharmaceutical Sciences. 2013;1:1-10.

8. Hanumanaik M, Patil U, Kumar G, Patel SK, Singh I, Jadatkar K. Design, evaluation and recent trends in transdermal drug delivery system: a review. International Journal of pharmaceutical sciences and research. 2012 Aug 1;3(8):2393.

9. Udupa N, Koteshwar KB. Vasantha kumar. Formulation and Evaluation of Captopril Transdermal preparations. Indian Drugs. 1992; 29(15): 680-850.

10. Kanig JL, Goodman H. Evaluative procedures for film-forming materials used in pharmaceutical applications. Journal of pharmaceutical sciences. 1962 Jan;51(1):77-83.

11. Amnuaikit C, Ikeuchi I, Ogawara KI, Higaki K, Kimura T. Skin permeation of propranolol from polymeric film containing terpene enhancers for transdermal use. International journal of pharmaceutics. 2005 Jan 31;289(1-2):167-78.

12. Sunil Reddy P, Sudhakar Babu K, Kumar N. Development and Validation of a Stability-Indicating RP-HPLC Method for the Simultaneous Estimation of Process Related Impurities and Degradation Products of Rasagiline Mesylate in Pharmaceutical Formulation. Journal of Chromatographic Science [Internet]. Oxford University Press (OUP); 2012 Sep 17;51(3):242-249.

13. Shelke PG, Chandewar AV. Validated High Performance Liquid Chromatographic Method for Determination of Rasagiline Mesylate under Oxidative, Photolytic and Thermal Stress Conditions. Int. J. Pharmtech Res. 2014;6:512-517.

14. Cilurzo F, Gennari CG, Minghetti P. Adhesive properties: a critical issue in transdermal patch development. Expert opinion on drug delivery. 2012 Jan 1;9(1):33-45.

15. Krishnaiah YS, Yang Y, Hunt RL, Khan MA. Cold flow of estradiol transdermal systems: Influence of drug loss on the in vitro flux and drug transfer across human epidermis. International journal of pharmaceutics. 2014 Dec 30;477(1-2):73-80.

16. Wokovich AM, Strasinger C, Kessler J, Cai B, Westenberger B, Rhee MJ, Raw A, Buhse LF. Cold flow measurement of transdermal drug delivery systems (TDDS). International Journal of Adhesion and Adhesives. 2015 Jun 1;59:71-76.

17. Krishnaiah YS, Yang Y, Hunt RL, Khan MA. Cold flow of estradiol transdermal systems: Influence of drug loss on the in vitro flux and drug transfer across human epidermis. International journal of pharmaceutics. 2014 Dec 30;477(1-2):73-80.

18. Thenge RR, Mahajan KG, Sawarkar HS, Adhao VS, Gangane PS. Formulation and evaluation of transdermal drug delivery system for lercanidipine hydrochloride. Int. J. Pharm. Tech. Res. 2010 Jan; 2(1):253-258.

19. Friend DR. In vitro skin permeation techniques. Journal of controlled release. 1992;18:235-248.

20. Abd E, Yousef SA, Pastore MN, Telaprolu K, Mohammed YH, Namjoshi S, Grice JE, Roberts MS. Skin models for the testing of transdermal drugs. Clinical pharmacology: advances and applications. 2016;8: 163.

21. Shah V, Raval S, Peer S, Upadhyay UM. A comparative evaluation of different membranes for their diffusion efficiency: An in vitro study. Pharma science monitor. 2010;1(2):41-49.

22. Lin SY, Lee CJ, Lin YY. Drug-polymer interaction affecting the mechanical properties, adhesion strength and release kinetics of piroxicam-loaded Eudragit E films plasticized with different plasticizers. Journal of controlled release. 1995 Mar 1;33(3):375381. 